\title{
STEADY STATES OF THE REACTION-DIFFUSION EQUATIONS. PART III: QUESTIONS OF MULTIPLICITY AND UN!QUENESS OF SOLUTIONSS
}

\author{
J. G. BURNELL ${ }^{1}$, A. A. LACEY ${ }^{2}$ and G. C. WAKE ${ }^{1}$
}

(Received 19 September 1983; revised 3 April 1984)

\begin{abstract}
In earlier papers (Parts I and II) existence and uniqueness of the solutions to a coupled pair of nonlinear elliptic partial differential equations with linear boundary conditions was considered. These equations arise when material is undergoing an exothermic chemical reaction which is sustained by the diffusion of a reactant. In this paper we establish the existence of multiple solutions for many different values of the parameters not considered in the earlier parts. It is shown that the case, also omitted in earlier parts, with perfect thermal and mass transfer on the boundary (the double-Dirichlet case) does have a unique solution for sufficiently large values of the exothermicity or an equivalent parameter. The methods of solution provide specific bounds on the region of existence of multiple solutions.
\end{abstract}

\section{Introduction}

In our two earlier papers (Burnell, Lacey and Wake [1, 2]) we investigated properties of the steady-state solutions of the equations governing the diffusion of a reactant which is undergoing an exothermic reaction. In Part I (Burnell, Lacey and Wake [1]) we showed this involved discussing properties of the solutions of the equations

with

$$
\begin{array}{ll}
\nabla^{2} u+\lambda(1+v) e^{u}=0 & \text { in } \Omega, \\
\nabla^{2} v-\lambda \alpha(1+v) e^{u}=0 & \text { in } \Omega, \\
\partial u / \partial n+\mu u=0 & \text { on } \partial \Omega, \\
\partial v / \partial n+\nu v=0 & \text { on } \partial \Omega,
\end{array}
$$

\footnotetext{
${ }^{1}$ Mathematics Department, Victoria University, Private Bag, Wellington, New Zealand. Please address all correspondence to Dr. Wake at this address.

${ }^{2}$ Mathematics Department, Heriot-Watt University, Edinburgh EH14 4AS, Scotland.

(C) Copyright Australian Mathematical Society 1985, Serial-fee code 0334-2700/85
} 
where $\Omega \subseteq \mathbf{R}^{n}$ is an open bounded set and $\lambda, \alpha, \mu, \nu$ are positive constants, $u$ is a dimensionless temperature rise over the ambient and $1+v$ is a dimensionless concentration of the diffusing reactant.

In Parts $\mathrm{I}$ and II we established the following properties.

THEOREM 1. (1) The equations (1), (2) have at least one solution for all values of the parameters, except when $\mu<\nu=\infty$. For the case $\mu<\nu=\infty$ and $n \leqslant 3$ there exists $\lambda^{*}$ (which depends on $\alpha$ and $\mu$ ) such that, for $\lambda<\lambda^{*},(1),(2)$ have at least one solution; and, for $\lambda>\lambda^{*}$, (1), (2) have no solutions (this holds for any $\alpha$ and any $\mu<\infty)$.

(2) For all cases, except when $\mu<\nu=\infty$, the solution to (1), (2) is unique for sufficiently small values of $\lambda$. Further, excluding the case $\mu=\nu=\infty$, the equations (1), (2) have a unique solution for sufficiently large values of $\lambda$.

(3) If $\mu=\nu$ then, for $\alpha$ sufficiently small, there are values of $\lambda$ for which (1), (2) have at least two solutions.

(4) Except for the case $\mu<\nu=\infty$ then, for any solution $(u, v)$ of (1), (2),

$$
\begin{array}{rlrl}
(\forall x \in \bar{\Omega}) & -1 & \leqslant v(x) & \leqslant 0, \\
(\forall x \in \bar{\Omega}) & 0 \leqslant u(x) \leqslant 1 / \alpha, & \text { for } \nu \leqslant \mu, \\
& 0 \leqslant u(x) \leqslant \nu / \alpha \mu, & \text { for } \mu<\nu<\infty .
\end{array}
$$

(5) If there is an interval $(a, b)$ such that, for $\lambda \in(a, b)$, the solution to (1), (2) is unique, then the solution branch $(\lambda,(u(\lambda), v(\lambda)))$ is a continuous function of $\lambda$ on $(a, b)$.

The purpose of this paper is to provide results regarding the multiplicity and uniqueness of solutions to (1) and (2) for those cases that were omitted in Parts I and II. Specifically we show that (1), (2) has multiple solutions for all $\mu, \nu<\infty$, when $\alpha$ is sufficiently small, and that the solution of (1), (2) when $\mu=\nu=\infty$ is unique for sufficiently large $\lambda$; and for a fixed $\lambda$, the solution is unique for sufficiently large $\alpha$. In the second case the equations (1), (2) are equivalent to a single nonlinear equation and, in fact, we prove in Section 3 a general uniqueness result for such an equation. Using the proofs of these results, in Section 5, we can obtain explicit bounds on the values of $\lambda$ for which multiple solutions exist.

\section{Results on multiple solutions}

In this section we shall discuss conditions under which the equations (1), (2) have multiple solutions for some appropriate (fixed) values of the parameters.

Firstly we shall show that when $\alpha$ is small, there are values of $\lambda$ for which there exist solutions of (1), (2) "near" the minimal solution for the case with $\alpha=0$. 
When $\alpha=0$, equations (1), (2) reduce to the single equation

$$
\begin{array}{ll}
\nabla^{2} u+\lambda e^{u}=0 & \text { in } \Omega, \\
\partial u / \partial n+\mu u=0 & \text { on } \partial \Omega .
\end{array}
$$

It is well known that the equations (3), (4) have solutions for values of $\lambda$ less than or equal to some $\lambda_{0}$ (see for instance Keller and Cohen [3]).

THEOREM 1. Suppose that, for $\lambda \leqslant \lambda_{0}, u_{\lambda}$ is the corresponding minimal solution to (3), (4). Then there exists $\alpha_{0}>0$ such that, for $\alpha \leqslant \alpha_{0}$ and $\lambda \leqslant \lambda_{0}$, (1), (2) has a solution $(u, v)$ with

$$
(\forall x \in \bar{\Omega}) \quad-1 \leqslant v(x) \leqslant 0,0 \leqslant u(x) \leqslant u_{\lambda}(x) .
$$

Proof. To prove this result we shall make use of Schauder's fixed point theorem. First, define a map $T: C(\bar{\Omega}) \times C(\bar{\Omega}) \rightarrow C(\bar{\Omega}) \times C(\bar{\Omega})$ by

$$
T(u, v)=\left(\lambda K_{\mu}(F(u, v)),-\lambda \alpha K_{\nu}(F(u, v))\right),
$$

where $K_{o}$ is the Green's function operator for $\left(-\nabla^{2}, \partial / \partial n+\sigma\right)$ and $F: C(\bar{\Omega}) \times$ $C(\bar{\Omega}) \rightarrow C(\bar{\Omega})$ is given by

$$
F(u, v)(x)=(1+v(x)) e^{u(x)} .
$$

In Part I of this paper (Burnell, Lacey and Wake [1]) we showed (see Section 2, Proposition 5) that if $(u, v)$ is a fixed point of $T$ then $(u, v)$ is a solution of (1), (2).

Now suppose $\lambda \leqslant \lambda_{0}$, then let

$D=\left\{(u, v) \in C(\bar{\Omega}) \times C(\bar{\Omega}):(\forall x \in \bar{\Omega}) 0 \leqslant u(x) \leqslant u_{\lambda}(x),-1 \leqslant v(x) \leqslant 0\right\}$. For $(u, v) \in D$, let $(w, z)=T(u, v)$. Then since $u_{\lambda}=\lambda K_{\mu}\left(e^{u_{\lambda}}\right)$,

$$
\left(w-u_{\lambda}\right)=\lambda K_{\mu}\left(F(u, v)-e^{u_{\lambda}}\right) .
$$

Also, since $-1 \leqslant v \leqslant 0$,

$$
\begin{aligned}
F(u, v)-e^{u_{\lambda}} & =(1+v) e^{u}-e^{u_{\lambda}} \\
& \leqslant(1+v) e^{u_{\lambda}}-e^{u_{\lambda}} \\
& =v e^{u_{\lambda}} . \leqslant 0 .
\end{aligned}
$$

Thus, as $K_{\mu}$ is a positive operator,

$$
w-u_{\lambda} \leqslant \lambda K_{\mu}(0)=0,
$$

so

$$
w \leqslant u_{\lambda}
$$

Since $(u, v) \in D,(1+v) e^{u} \geqslant 0$; hence by the maximum principle, $w \geqslant 0$. Therefore $(\forall x \in \bar{\Omega}), 0 \leqslant w(x) \leqslant u_{\lambda}(x)$. 
Also, it is clear that $z \leqslant 0$ and

$$
\|z\|_{0}=\left\|\lambda \alpha K_{\nu}(F(u, v))\right\|_{0} \leqslant \lambda \alpha\left\|K_{\nu}\right\|_{0}\|F(u, v)\|_{0}
$$

where $\left\|K_{\nu}\right\|_{0}$ is the norm of the operator $K_{\nu}$ (with respect to the supremum norm on $C(\bar{\Omega})$ ). Now

$$
\begin{aligned}
\|F(u, v)\|_{0} & =\sup _{x \in \Omega}\left|(1+v(x)) e^{u(x)}\right| \\
& \leqslant \sup _{x \in \Omega}\left|e^{u_{\lambda}(x)}\right|=\left\|e^{u_{\lambda}}\right\|_{0} .
\end{aligned}
$$

Hence $\|z\|_{0} \leqslant \lambda \alpha\left\|K_{v}\right\|_{0}\left\|e^{u_{\lambda}}\right\|_{0}$. It is known (Keller and Cohen [3]) that if $U_{0}$ is the minimal solution of (3), (4) when $\lambda=\lambda_{0}$ then, for $\lambda \leqslant \lambda_{0}$, we have $u_{\lambda} \leqslant U_{0}$.

So, if $\alpha_{0}$ is chosen so that

$$
\lambda_{0} \alpha_{0}\left\|K_{\nu}\right\|_{0}\left\|e^{U_{0}}\right\|_{0}<1
$$

then, for $\alpha \leqslant \alpha_{0}, \lambda \leqslant \lambda_{0}$,

$$
\|z\|_{0}<1
$$

Therefore

$$
(\forall x \in \bar{\Omega})-1 \leqslant z(x) \leqslant 0 .
$$

This then means that, for $\alpha \leqslant \alpha_{0}$ and $\lambda \leqslant \lambda_{0}, T(D) \subseteq D$.

Now, it is clear that $D$ is a closed convex subset of $C(\bar{\Omega}) \times C(\bar{\Omega})$. Also, using the known properties of $K_{\mu}$ and $K_{v}$ it is easily shown (see Burnell, Lacey and Wake [1]) that $T$ is a compact map. Hence the Schauder fixed point theorem ensures that there exists $(u, v) \in D$ such that

$$
T(u, v)=(u, v) \text {, }
$$

when $\alpha \leqslant \alpha_{0}$ and $\lambda \leqslant \lambda_{0}$. Consequently, for $\alpha \leqslant \alpha_{0}$ and $\lambda \leqslant \lambda_{0}$, there exists a solution $(u, v)$ of $(1),(2)$ with

$$
(\forall x \in \bar{\Omega}) \quad 0 \leqslant u(x) \leqslant u_{\lambda}(x),-1 \leqslant v(x) \leqslant 0 .
$$

In order to prove that the equations (1), (2) have at least 2 solutions for certain values of the parameters we make use of properties of the Leray-Schauder degree and some results from Part I (Burnell, Lacey and Wake [1]). For easy reference we shall summarise these results here.

THEOREM 2. (1) Suppose $X$ is a normed vector space and $D$ is an open bounded subset of $X$ containing 0 . Further, suppose $S: \bar{D} \rightarrow X$ is a compact map with $0 \notin(I-S)(\partial D)$. Then the Leray-Schauder degree of $I-S$ at 0 on $D$ is an integer which satisfies:

(a) if $S=0$ then $d(I, D, 0)=1$;

(b) if $d(I-S, D, 0) \neq 0$ then there exists $x \in D$ such that $x-S x=0$; 
(c) if $H: \bar{D} \times[0,1] \rightarrow X$ satisfies:

(i) $(\forall s \in[0,1]) H(\cdot, s): \bar{D} \rightarrow X: x \rightarrow H(x, s)$ is compact;

(ii) $(\forall \varepsilon>0)(\exists \delta>0)$ such that whenever $\left|s_{1}-s_{2}\right|<\delta$,

$$
(\forall x \in \bar{D})\left\|H\left(x, s_{1}\right)-H\left(x, s_{2}\right)\right\|<\varepsilon ;
$$

(iii) $(\forall s \in[0,1]) 0 \notin(I-H(\cdot, s))(\partial D)$, then $d(I-H(\cdot, s), D, 0)$ is independent of $s \in[0,1]$

(d) If $E \subseteq D$ is closed and $0 \notin(I-S)(E)$ then

$$
d(I-S, D, 0)=d(I-S, D \backslash E, 0) .
$$

(2) If $T$ is the map defined by (5), and $\mu, \nu<\infty$ then, for any $\alpha>0$ and $\lambda \geqslant 0, T$ is compact and

$$
d(I-T, B(2+m(\alpha)), 0)=1,
$$

where

$$
m(\alpha)= \begin{cases}1 / \alpha, & \text { for } \mu \geqslant \nu \\ \nu /(\alpha \mu), & \text { for } \mu<\nu\end{cases}
$$

and

$$
B(r)=\left\{(w, z) \in C(\bar{\Omega}) \times C(\bar{\Omega}):\|w\|_{0}+\|z\|_{0}<r\right\} .
$$

(3) Suppose $\mu, \nu<\infty$. Then, for sufficiently large values of $\lambda$, (1), (2) has a unique solution $(u, v)$ and for some constant $A$, independent of $\lambda$,

$$
\begin{gathered}
(\forall x \in \bar{\Omega})\left(\nu / \mu-A(\lambda \alpha)^{-1 / 2}\right) / \alpha \leqslant u(x) \leqslant 1 / \alpha \quad \text { if } \mu \geqslant \nu, \\
\left(1-A(\lambda \alpha)^{-1 / 2}\right) \nu /(\alpha \mu) \leqslant u(x) \leqslant \nu /(\alpha \mu) \text { if } \mu<\nu .
\end{gathered}
$$

In order to define the Leray-Schauder degree of $I-T$ we need to find an open set $D \subseteq C(\bar{\Omega}) \times C(\bar{\Omega})$ with $0 \notin(I-T)(\partial D)$. Further we would like to show that there is a solution to (1), (2) in $D$ which is different from the solution that exists as a consequence of Theorem 1. This is the purpose of the next result. For the rest of this section let $u_{\lambda}$ be the solution to (3), (4) defined in Theorem 1 .

Proposition 3. Fix $\lambda_{1}$ so that $0<\lambda_{1}<\lambda_{0}$ and fix $\mu, \nu<\infty$. Then there exist constants $C, \alpha_{1}$, and a function w such that, for $\lambda_{1}<\lambda$ and $0<\alpha<\alpha_{1}$ : whenever $(u, v)$ is a solution of (1), (2) with

$$
(\forall x \in \bar{\Omega}) \quad C w(x) \leqslant u(x),
$$

we have

$$
(\forall x \in \bar{\Omega}) \quad C w(x)<u(x)
$$

and

$$
\left\|u_{\lambda}\right\|_{0}<C\|w\|_{0}
$$


Proof. Here we will only present the proof for the case when $\mu>\nu$, and will discuss the case $\mu \leqslant \nu$ at the end of this proof.

Choose a nonnegative function $f \in C^{2}(\bar{\Omega})$ which has its nonempty support in $\Omega$. Let $w$ be the unique (positive) solution of

$$
\left.\begin{array}{ll}
\nabla^{2} w+f=0 & \text { in } \Omega \\
\frac{\partial w}{\partial n}+\mu w=0 & \text { on } \partial \Omega .
\end{array}\right\}
$$

Choose $C$ so that it satisfies the inequalities

$$
(\forall x \in \bar{\Omega}) \frac{\nu}{\mu} e^{C w(x)}-\frac{C f(x)}{\lambda_{1}} \geqslant 1,
$$

and, for $\lambda_{1} \leqslant \lambda \leqslant \lambda_{0}$,

$$
C\|w\|_{0}>\left\|u_{\lambda}\right\|_{0}
$$

Since $\left(\forall \lambda \in\left[\lambda_{1}, \lambda_{0}\right]\right) u_{\lambda} \leqslant u_{\lambda_{0}}$ on $\bar{\Omega}$, and $(\forall x \in \bar{\Omega}) w(x) \geq 0$, it is easily seen that such a choice of $C$ is possible. Also, choose $\alpha_{1}$ so that, on $\bar{\Omega}$,

$$
\alpha_{1} C w e^{C w}<1 \text {. }
$$

Then it follows that

$$
C w<1 / \alpha_{1} \text {. }
$$

Now, suppose $\lambda>\lambda_{1}, \alpha \in\left(0, \alpha_{1}\right]$ and $(u, v)$ is a solution of (1), (2) with $(\forall x \in \bar{\Omega}) C w(x) \leqslant u(x)$. Then $h=\alpha u+v$ satisfies the equation

$$
\left.\begin{array}{ll}
\nabla^{2} h=0 & \text { in } \Omega, \\
\frac{\partial h}{\partial n}+\mu h=(\mu-\nu) v & \text { on } \partial \Omega .
\end{array}\right\}
$$

Since $(\forall x \in \bar{\Omega})-1 \leqslant v(x) \leqslant 0$, it follows from the maximum principle that

$$
(\forall x \in \bar{\Omega}) \quad-1+\nu / \mu \leqslant h(x) \leqslant 0 .
$$

Using (1) we then see that $\varphi=u$ is the unique solution of

$$
\left.\begin{array}{ll}
\nabla^{2} \varphi+\lambda(1+h-\alpha \varphi) e^{u}=0 & \text { in } \Omega, \\
\frac{\partial \varphi}{\partial n}+\mu \varphi=0 & \text { on } \partial \Omega .
\end{array}\right\}
$$

We shall now show that $C w$ is a strict lower solution for the equation (14):

$$
\begin{aligned}
\nabla^{2}(C w)+\lambda(1+h-\alpha C w) e^{u} & =-C f+\lambda(1+h-\alpha C w) e^{u} \\
& \geqslant \lambda\left[-C f / \lambda+\left(\nu / \mu-\alpha_{1} C w\right) e^{C w}\right] \\
& \geqslant \lambda\left[-C f / \lambda_{1}+(\nu / \mu) e^{C w}-\alpha_{1} C w e^{C w}\right] \\
& >\lambda[1-1]=0 \quad \text { in } \Omega .
\end{aligned}
$$


Also,

$$
\frac{\partial(C w)}{\partial n}+\mu(C w)=0 \text { on } \partial \Omega
$$

Hence $C w$ is a strict lower solution for (14). Now

$$
\nabla^{2}(1 / \alpha)+\lambda(1+h-\alpha(1 / \alpha)) e^{u}=\lambda h e^{u} \leqslant 0 \quad \text { in } \Omega,
$$

and

$$
\frac{\partial(1 / \alpha)}{\partial n}+\mu(1 / \alpha)=\mu / \alpha>0 \text { on } \partial \Omega,
$$

so $\varphi(x)=1 / \alpha$ is an upper solution for (14). It then follows from (11) and the theory of upper and lower solutions that the unique solution $\varphi=u$ of (14) satisfies

$$
(\forall x \in \bar{\Omega}) \quad C w(x)<u(x)<1 / \alpha .
$$

For the case when $\mu \leqslant \nu$ the proof is essentially the same. The differences are:

$$
\text { (13) becomes }(\forall x \in \bar{\Omega}) \quad 0 \leqslant h(x) \leqslant \nu / \mu-1 \text {; }
$$

(8) becomes $e^{C w}-C f / \lambda_{1} \geqslant 1$;

(c) $\quad \varphi(x)=\nu /(\alpha \mu)$ is used as an upper solution for (14).

This then leads us to our main result concerning multiple solutions.

THeOrem 4. Suppose $\mu, \nu<\infty$ and $\lambda_{1} \in\left(0, \lambda_{0}\right)$. Then there exists $\alpha_{2}>0$ such that, for $\alpha \in\left(0, \alpha_{2}\right]$ and $\lambda \in\left[\lambda_{1}, \lambda_{0}\right],(1),(2)$ has at least two distinct solutions.

Proof. We present the proof for the case when $\mu \geqslant \nu$ only, as the proof for the other case, $\mu<\nu$, is similar and is left to the reader. Let $C, w$ be as in Proposition 3 ((7), (8) and (9)), and choose $\alpha_{2}$ so that it satisfies

$$
\left.\begin{array}{c}
\alpha_{2} \leqslant \min \left\{\alpha_{0}, \alpha_{1}\right\}, \\
\text { and }(\forall x \in \bar{\Omega}) \quad \alpha_{2} C w(x)<\frac{1}{2}(\nu / \mu),
\end{array}\right\}
$$

where $\alpha_{0}$ and $\alpha_{1}$ are given in Theorem 1 and Proposition 3 respectively.

Now, by Theorem 2(3), there exists $\lambda_{2}>\lambda_{0}$ such that (1), (2) has a unique solution $(U, V)$ when $\lambda=\lambda_{2}$ and $\alpha=\alpha_{2}$, and

$$
\left.\begin{array}{cc}
(\forall x \in \bar{\Omega}) & U(x) \geqslant\left(\nu / \mu-A\left(\lambda_{2} \alpha_{2}\right)^{-1 / 2}\right) / \alpha_{2} \\
\text { so }(\forall x \in \bar{\Omega}) & \left(\nu / \mu-A\left(\lambda_{2} \alpha_{2}\right)^{-1 / 2}\right) / \alpha_{2}>C w(x) .
\end{array}\right\}
$$

(This last inequality follows from (15), by choosing $\lambda_{2}$ large enough.) Fix $\alpha \in\left(0, \alpha_{2}\right]$ and $\lambda \in\left[\lambda_{1}, \lambda_{0}\right]$, and set

$$
\begin{aligned}
& B=\{(u, v) \in C(\bar{\Omega}) \times C(\bar{\Omega}): \\
&(\forall x \in \bar{\Omega}) C w(x)<u(x)<1 / \alpha+1, \text { and }-2<v(x)<1\} .
\end{aligned}
$$


Now, it is clear that $B$ is an open subset of $C(\bar{\Omega}) \times C(\bar{\Omega})$ and

$$
\begin{aligned}
\bar{B}=\{(u, v) & \in C(\bar{\Omega}) \times C(\bar{\Omega}): \\
& (\forall x \in \bar{\Omega}) C w(x) \leqslant u(x) \leqslant 1 / \alpha+1 \text { and }-2 \leqslant v(x) \leqslant 1\} .
\end{aligned}
$$

Let $T$ be the map defined by (5) and suppose $H: \bar{B} \times[0,1] \rightarrow C(\bar{\Omega}) \times C(\bar{\Omega})$ is the homotopy given by

$$
H((u, v), t)=\left(t+(1-t) \lambda_{2} / \lambda\right) T(u, v) .
$$

We shall now show that $H$ satisfies the conditions of Theorem 2(1)(c). First, it is clear that, for each $t \in[0,1], H(\cdot, t)$ is compact since $T$ is a compact map (see Theorem 1 of Section 1). Also as $\bar{B}$ is a bounded set, there exists a constant $M$ such that

$$
(\forall(u, v) \in \bar{B}) \quad\|T(u, v)\|_{0} \leqslant M .
$$

Hence

$$
\begin{aligned}
(\forall t, s \in[0,1]),(\forall(u, v) & \in \bar{B})\|H((u, v), t)-H((u, v), s)\|_{0} \\
& =\left|t-s\left\|1-\lambda_{2} / \lambda\left|\|T(u, v)\|_{0} \leqslant M\right| 1-\lambda_{2} / \lambda|| t-s \mid .\right.\right.
\end{aligned}
$$

So, it is easily seen that conditions (i) and (ii) of Theorem $2(1)(c)$ are satisfied.

Now, as noted in Theorem 1 , if $(u, v)$ is a fixed point of $T$ then $(u, v)$ satisfy (1), (2). Similarly, if $H((u, v), t)=(u, v)$ for some $t \in[0,1]$ then $(u, v)$ satisfy

$$
\left.\begin{array}{cc}
\nabla^{2} u+\left(\lambda t+(1-t) \lambda_{2}\right)(1+v) e^{u}=0 & \text { in } \Omega, \\
\nabla^{2} v-\left(\lambda t+(1-t) \lambda_{2}\right) \alpha(1+v) e^{u}=0 & \text { in } \Omega,
\end{array}\right\}
$$

Suppose, for some $t \in[0,1]$ and $(u, v) \in \bar{B}, H((u, v), t)=(u, v)$. Then, since $\lambda t+(1-t) \lambda_{2} \in\left[\lambda_{1}, \lambda_{2}\right]$ and

$$
(\forall x \in \bar{\Omega}) \quad C w(x) \leqslant u(x),
$$

it follows from Proposition 3 that

$$
(\forall x \in \bar{\Omega}) \quad C w(x)<u(x) .
$$

Also, by Section 1, Theorem 1(4),

$$
(\forall x \in \bar{\Omega}) \quad u(x) \leqslant 1 / \alpha,-1 \leqslant v(x) \leqslant 0 .
$$

Hence $(u, v) \in B$.

Consequently, we see that, for $t \in[0,1]$,

$$
(0,0) \notin(I-H(\cdot, t))(\partial B) .
$$

So, the conditions of Theorem 2(1)(c) are satisfied. Therefore

$$
\begin{aligned}
d(I-T, B, 0) & =d(I-H(\cdot, 1), B, 0) \\
& =d(I-H(\cdot, 0), B, 0) .
\end{aligned}
$$


Now, for $(u, v) \in \dot{B}$,

$$
H((u, v), 0)=\left(\lambda_{2} K_{\mu}\left((1+v) e^{u}\right),-\lambda_{2} \alpha K_{\nu}\left((1+v) e^{u}\right)\right) .
$$

So the fixed points of $H(\cdot, 0)$ are the solutions of (1), (2) with $\lambda=\lambda_{2}$. Then it follows from (16) and Theorem $1(4)$ that $(U, V) \in B$. Also, by Theorem 2(1)(d), (2),

$$
d(I-H(\cdot, 0), B, 0)=d(I-H(\cdot, 0), B(2+m(\alpha)), \overline{0})=1,
$$

and, so, by Theorem $3(1)(\mathrm{b})$, there exists $\left(u_{1}, v_{1}\right) \in B$ which satisfy equation (1), (2).

Since $\alpha_{2} \leqslant \alpha_{0}$ and $\lambda \leqslant \lambda_{0}$, it follows from Theorem 1 that there exists a solution $\left(u_{2}, v_{2}\right)$ of (1), (2) such that

$$
(\forall x \in \bar{\Omega}) \quad u_{2}(x) \leqslant u_{\lambda}(x) .
$$

Then, as $\left(u_{1}, v_{1}\right) \in B$, it follows from (9) that

$$
(\forall x \in \bar{\Omega}) \quad\left\|u_{2}\right\|_{0} \leqslant\left\|u_{\lambda}\right\|_{0}<C\|w\|_{0} \leqslant\left\|u_{1}\right\|_{0} .
$$

Hence $u_{1} \neq u_{2}$ and $\left(u_{1}, v_{1}\right)$ and $\left(u_{2}, v_{2}\right)$ are distinct solutions of (1), (2). Q.E.D.

\section{Uniqueness of solutions for large $\lambda$}

As stated in Section 1, Theorem 1, we investigated in Part II (Burnell, Lacey and Wake [2]) situations for which the equations (1), (2) will have a unique solution. Since completing that paper we have succeeded in extending the results given there. It is the purpose of the next two sections to look at these extensions. In particular we shall prove that if $\mu=\nu=\infty$ then (1), (2) have a unique solution for sufficiently large values of $\lambda$; and if $\alpha$ is large enough then (1), (2) will have a unique solution for all values of the other parameters except when $\mu<\nu=\infty$.

Firstly we shall look at the case when $\mu=\nu=\infty$. Here we see that the harmonic function $h=\alpha u+v$ is zero and (1), (2) reduce to the single equation

$$
\left.\begin{array}{rr}
\nabla^{2} u+\lambda(1-\alpha u) e^{u}=0 & \text { in } \Omega, \\
u=0 & \text { on } \partial \Omega .
\end{array}\right\}
$$

In fact we shall consider the more general equation

$$
\left.\begin{array}{rr}
\nabla^{2} u+\lambda f(u)=0 & \text { in } \Omega, \\
u=0 & \text { on } \partial \Omega,
\end{array}\right\}
$$

where $f: \mathbf{R} \rightarrow \mathbf{R}: t \mapsto(1-t) g(t)$ and $g: \mathbf{R} \rightarrow \mathbf{R}$ satisfies

(a) $(\forall t \in[0,1]) 1 \geqslant g(t) \geqslant \gamma_{0}>0$,

(b) $g$ is a $C^{2}$ function.

(c) $(\forall t \in \mathbf{R}) g(t)>0$. 
Now, it follows easily from the maximum principle that any solution of (21), $u$ say, satisfies

$$
(\forall x \in \bar{\Omega}) \quad 0 \leqslant u(x) \leqslant 1 .
$$

Further, $u=0$ and $u(x)=1$ are lower and upper solutions of (21) respectively; hence, for any $\lambda \geqslant 0,(21)$ has at least one solution. The first step in showing that (21) has a unique solution for sufficiently large values of $\lambda$ is to find bounds on the solution of (21) when $\lambda$ is large. For this purpose, we require properties of the solution of the following initial value problem,

$$
\left.\begin{array}{l}
z^{\prime \prime}+f(z)=0 \text { on }(0, \infty) \\
z(0)=0, \quad z^{\prime}(0)=\sqrt{2 \int_{0}^{1} f(t) d t} .
\end{array}\right\}
$$

Proposition 1. Equation (22) has a unique solution $z$, and $(\forall \mathrm{s} \in[0, \infty))$

$$
1-\exp \left(-\sqrt{\gamma_{0}} s\right) \leqslant z(s)<1
$$

Proof. Now, (22) has at most one solution. Suppose (22) has a solution $z$; then

$$
z^{\prime}(s)^{2}=z^{\prime}(0)^{2}-2 \int_{0}^{z(s)} f(t) d t .
$$

If there exists $s_{1} \in(0, \infty)$ for which $z\left(s_{1}\right)=1$ then (from (23)) $z^{\prime}\left(s_{1}\right)=0$. Hence, on $\left[0, s_{1}\right], z$ satisfies

$$
\begin{gathered}
z^{\prime \prime}+f(z)=0, \\
z\left(s_{1}\right)=1, \quad z^{\prime}\left(s_{1}\right)=0 ;
\end{gathered}
$$

but the function $z_{1}(s)=1$ is the unique solution of this equation. It then follows from (23) that $z^{\prime}(s)>0$ on $(0, \infty)$, and so we must have

$$
(\forall s \in[0, \infty)) \quad 0 \leqslant z(s)<1 \text {. }
$$

This then means that (22) is equivalent to the problem

$$
\begin{gathered}
z^{\prime \prime}+f_{1}(z)=0 \text { on }(0, \infty), \\
z(0)=0, \quad z^{\prime}(0)=\sqrt{2 \int_{0}^{1} f(s) d s,}
\end{gathered}
$$

where $f_{1}$ is a suitable bounded function which agrees with $f$ on $[0,1]$. And, from the theory of ordinary differential equations, we know that this problem has a solution defined on $[0, \infty)$.

Thus we have shown that (22) has a unique solution $z$ which satisfies $(\forall s \in$ $[0, \infty)) 0 \leqslant z(s)<1$. Therefore, $(\forall s \in(0, \infty)) z^{\prime \prime}(s)<0$; so $z^{\prime}$ is decreasing on $(0, \infty)$. Clearly this means that $z^{\prime}(s) \rightarrow 0$ as $s \rightarrow \infty$. It then follows from (23) that $z(s) \rightarrow 1$ as $s \rightarrow \infty$. 
Consider the boundary-value problem

$$
\begin{aligned}
\varphi^{\prime \prime}+(1-\varphi) g(z) & =0 & \text { in }(0, \infty), \\
\varphi(0)=0, \quad \varphi(s) & \rightarrow 1 & \text { as } s \rightarrow \infty ;
\end{aligned}
$$

clearly $\varphi=z$ is the unique solution of this problem. If we take the function $\varphi_{1}(s)=1-\exp \left(-\sqrt{\gamma_{0}} s\right)$ then

$$
\varphi_{1}^{\prime \prime}+\left(1-\varphi_{1}\right) g(z)=\left(-\gamma_{0}+g(z)\right) \exp \left(-\sqrt{\gamma_{0}} s\right)>0
$$

and

$$
\varphi_{1}(0)=0, \quad \varphi_{1}(s) \rightarrow 1 \text { as } s \rightarrow \infty .
$$

Hence $\varphi_{1}$ is a lower solution for this problem. Similarly $\varphi_{2}(s)=1$ is an upper solution; therefore we must have

$$
(\forall s \in[0, \infty)) \quad 1-\exp \left(-\sqrt{\gamma_{0}} s\right) \leqslant z(s)<1 .
$$

Now $\partial \Omega$ is of class $C^{2+o}$, hence $\partial \Omega$ satisfies a uniform interior sphere condition. That is, there exists $\rho>0$ such that, for each $x \in \partial \Omega$, there is an open ball of radius $\rho, B_{x}$, such that $B_{x} \subseteq \Omega$ and $x \in \partial B_{x}$. Then we have:

THEOREM 2. Suppose $u$ is a solution of (21); then there exist constants $A, B$ such that, for $\lambda$ sufficiently large,

$$
\begin{gathered}
1-\exp \left(-A \lambda^{1 / 2}\right) \leqslant u(x) \leqslant 1 \text { when } d(x, \partial \Omega) \geqslant \rho / 2, \\
z\left(\lambda^{1 / 2} y\right)-B / \lambda^{1 / 2} \leqslant u(x) \leqslant z\left(\lambda^{1 / 2} y\right)+B / \lambda^{1 / 2} \text { when } y=d(x, \partial \Omega) \leqslant \rho / 2,
\end{gathered}
$$

where $z$ is the unique solution of (22) and $d(x, \partial \Omega)$ is the distance from $x$ to the boundary $\partial \Omega$.

Proof. The theorem is proved by constructing suitable upper and lower solutions for the equation

$$
\left.\begin{array}{rlrl}
\nabla^{2} \varphi+\lambda(1-\varphi) g(u) & =0 & & \text { in } \Omega, \\
\varphi & =0 & & \text { on } \partial \Omega,
\end{array}\right\}
$$

where $u$ is a solution of (21).

Choose $k>0$ so that the solution $w$ of

$$
\begin{array}{rlrl}
\nabla^{2} w+k=0 & & \text { in } \Omega, \\
w & =0 & & \text { on } \partial \Omega,
\end{array}
$$

satisfies $(\forall x \in \bar{\Omega})|\nabla w(x)| \leqslant \gamma_{0} / 2$. Let us now show that $u_{1}=1-\exp \left[-\lambda^{1 / 2} w\right]$ is a lower solution for (24) for $\lambda$ sufficiently large:

$$
\begin{aligned}
\nabla^{2} u_{1}+\lambda\left(1-u_{1}\right) g(u) & =\sqrt{\lambda}\left(\nabla^{2} w\right) e^{-\sqrt{\lambda} w}-\lambda|\nabla w|^{2} e^{-\sqrt{\lambda} w}+\lambda e^{-\sqrt{\lambda} w} g(u) \\
& =\sqrt{\lambda} e^{-\sqrt{\lambda} w}\left[-k-\sqrt{\lambda}|\nabla w|^{2}+\sqrt{\lambda} g(u)\right] \\
& >\sqrt{\lambda} e^{-\sqrt{\lambda} w}\left[-k-\gamma_{0} \sqrt{\lambda} / 2+\gamma_{0} \sqrt{\lambda}\right] \\
& >0
\end{aligned}
$$


if $\sqrt{\lambda}>2 k / \gamma_{0}$. Also $u_{1}=0$ on $\partial \Omega$; hence $u_{1}$ is a lower solution for (24) if $\lambda>4 k^{2} / \gamma_{0}^{2}$.

Now, $u_{2}(x)=1$ is an upper solution for (24) and $(\forall x \in \bar{\Omega}) u_{1}(x)<u_{2}(x)$. Therefore (24) has a solution $\varphi$ which satisfies $(\forall x \in \bar{\Omega}) u_{1}(x) \leqslant \varphi(x) \leqslant 1$, if $\lambda>4 k^{2} / \gamma_{0}^{2}$. Since $\varphi=u$ is the unique solution of (24), we find that, for $\lambda>4 k^{2} / \gamma_{0}^{2}$,

$$
(\forall x \in \bar{\Omega}) \quad 1-\exp \left(-\lambda^{1 / 2} w(x)\right) \leqslant u(x) \leqslant 1 .
$$

If $\Omega^{\prime}=\{x \in \Omega: d(x, \partial \Omega) \geqslant \rho / 2\}$ then $\Omega^{\prime}$ is closed and bounded. Also, it follows from the maximum principle that there exists a constant $A$ such that

$$
(\forall x \in \bar{\Omega}) \quad w(x) \geqslant A>0 .
$$

Thus, when $\lambda>4 k^{2} / \gamma_{0}^{2}$,

$$
1-\exp \left(-A \lambda^{1 / 2}\right) \leqslant u(x) \leqslant 1 \text { if } d(x, \partial \Omega) \geqslant \rho / 2 .
$$

Now, let us find upper and lower solutions which allow us to show that the second inequality holds. Let $T_{\rho}=\{x \in \Omega: d(x, \partial \Omega)<\rho / 2\}$; then $T_{\rho}$ is open and bounded. We define a function $w$ on $T_{\rho}$ as follows:

$$
w(x)=z\left(\lambda^{1 / 2} d(x, \partial \Omega)\right) .
$$

We then see that on $T_{\rho}$,

$$
\nabla^{2} w(x)=\lambda z^{\prime \prime}\left(\lambda^{1 / 2} y\right)+\lambda^{1 / 2}(\nabla \cdot \eta) z^{\prime}\left(\lambda^{1 / 2} y\right),
$$

where $y=d(x, \partial \Omega)$ and $\eta$ is the unit normal vector to the surface $\{y: d(y, \partial \Omega)=$ $d(x, \partial \Omega)\}$. Since $\partial \Omega$ is of class $C^{2+\sigma}$ there exists a constant $M$ such that

$$
\left(\forall x \in T_{\rho}\right) \quad|\nabla \cdot \eta(x)| \leqslant M .
$$

We now show that the function $w(x)-B / \lambda^{1 / 2}$ is a lower solution for the equation

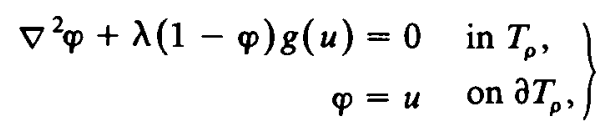

if $\lambda$ is sufficiently large.

Using properties of $z$ from Proposition 1 we have, on $T_{\rho}$,

$$
\begin{aligned}
\nabla^{2}(w- & \left.B / \lambda^{1 / 2}\right)+\lambda\left(1-\left(w-B / \lambda^{1 / 2}\right)\right) g(u) \\
= & \lambda z^{\prime \prime}\left(\lambda^{1 / 2} y\right)+\lambda^{1 / 2}(\nabla \cdot \eta) z^{\prime}\left(\lambda^{1 / 2} y\right)+\lambda\left(1-z\left(\lambda^{1 / 2} y\right)+B / \lambda^{1 / 2}\right) g(u) \\
= & \lambda\left(1-z\left(\lambda^{1 / 2} y\right)\right)\left[g(u)-g\left(z\left(\lambda^{1 / 2} y\right)\right)\right] \\
& +\lambda^{1 / 2}(\nabla \cdot \eta) z^{\prime}\left(\lambda^{1 / 2} y\right)+\lambda^{1 / 2} B g(u) \\
\geqslant & \lambda^{1 / 2}\left[-\lambda^{1 / 2} \exp \left(-\left(\gamma_{0} \lambda\right)^{1 / 2} y\right)\left|g(u)-g\left(z\left(\lambda^{1 / 2} y\right)\right)\right|-M z^{\prime}(0)+B \gamma_{0}\right] .
\end{aligned}
$$


Now, since $g(u)-g\left(z\left(\lambda^{1 / 2} y\right)\right)$ is a bounded function of $x$ and, when $y=$ $d(x, \partial \Omega)=0, g(u)-g\left(z\left(\lambda^{1 / 2} y\right)\right)=0$, it follows that there exists a constant $M_{1}$ with $\lambda^{1 / 2} \exp \left(-\left(\gamma_{0} \lambda\right)^{1 / 2} y\right)\left|g(u)-g\left(z\left(\lambda^{1 / 2} y\right)\right)\right|<M_{1}$ for sufficiently large $\lambda$ and for $x \in T_{p}$. Thus if $B=\left(M_{1}+M z^{\prime}(0)\right) / \gamma_{0}$ then for $\lambda$ sufficiently large,

$$
\nabla^{2}\left(w-B / \lambda^{1 / 2}\right)+\lambda\left(1-\left(w-B / \lambda^{1 / 2}\right)\right) g(u) \geqslant 0 \text { on } T_{\rho} .
$$

Âiso, when $x \in \hat{\partial} T_{\rho}$ we either have

(a) $d(x, \partial \Omega)=0$ in which case $w(x)-B / \lambda^{1 / 2}=z(0)-B / \lambda^{1 / 2}=-B / \lambda^{1 / 2}<$ $u(x)$, since $u \geqslant 0$ on $\bar{\Omega}$; or

(b) $d(x, \partial \Omega)=\rho / 2$ in which case, if $\lambda^{1 / 2} e^{-A \lambda^{1 / 2}}<B$ and $\lambda>4 k^{2} / \gamma_{0}^{2}$,

$$
\begin{aligned}
w(x)-B / \lambda^{1 / 2} & =z\left(\lambda^{1 / 2} \rho / 2\right)-B / \lambda^{1 / 2} \\
& <1-B / \lambda^{1 / 2} \quad \text { by Proposition } 1 \\
& <1-e^{-A \lambda^{1 / 2}} \\
& \leqslant u(x) \text { by }(25) .
\end{aligned}
$$

Therefore, when $\lambda$ is sufficiently large, $w-B / \lambda^{1 / 2}$ is a lower solution for (27).

A similar argument shows that the function $w(x)+B / \lambda^{1 / 2}$ is an upper solution for (27) when $\lambda$ is sufficiently large. Consequently, the unique solution $\varphi=u$ of (27) must satisfy

$$
\left(\forall x \in T_{\rho}\right) \quad z\left(\lambda^{1 / 2} y\right)-B / \lambda^{1 / 2} \leqslant u(x) \leqslant z\left(\lambda^{1 / 2} y\right)+B / \lambda^{1 / 2},
$$

where $y=d(x, \partial \Omega)$.

We shall later require the following result. For the rest of this paper we shall let $z$ be the unique solution of (22).

LEMMA 3. Suppose $\psi$ is the unique solution of

$$
\left.\begin{array}{rlrl}
\frac{d^{2} \psi}{d s^{2}}+f^{\prime}(z(s)) \psi & =0, & & s \in(0, \infty), \\
\psi(0) & =0, & & \psi^{\prime}(0)=1 .
\end{array}\right\}
$$

Then there exists $X>0$ and $\Pi_{1}<0$ such that

$$
\psi^{\prime}(X)>0
$$

and $(\forall s \geqslant X) f^{\prime}(z(s)) \leqslant \Pi_{1}$

Proof. Firstly, the unique solution of (29) is

$$
\psi(s)=z^{\prime}(0) z^{\prime}(s) \int_{0}^{s} \frac{d t}{z^{\prime}(t)^{2}} .
$$


Also, using (22), (23) and Proposition 1

$$
\begin{aligned}
z^{\prime}(s)^{2} & =2 \int_{z(s)}^{1} f(t) d t \leqslant 2 \int_{1-\exp \left(-\sqrt{\gamma_{0}} s\right)}^{1} f(t) d t \\
& \leqslant 2 \exp \left(-\sqrt{\gamma_{0}} s\right) .
\end{aligned}
$$

Hence

$$
\int_{0}^{s} \frac{d t}{z^{\prime}(t)^{2}} \geqslant \frac{1}{2 \sqrt{\gamma_{0}}}\left(\exp \left(\sqrt{\gamma_{0}} s\right)-1\right) \rightarrow \infty \quad \text { as } s \rightarrow \infty .
$$

Accordingly, using L'Hôpital's rule on the above gives that $\psi(s) \rightarrow \infty$ as $s \rightarrow \infty$. The result then follows from the properties of $z$ given in Proposition 1. Q.E.D.

The approach that we now take is to assume that (21) does not have a unique solution for some value of $\lambda$, and to then show that this leads to a contradiction if $\lambda$ is sufficiently large. If (21) does not have a unique solution then the minimal and maximal solutions of (21), $u_{m}$ and $u_{M}$ respectively must satisfy (see Keller and Cohen [3])

$$
(\forall x \in \bar{\Omega}) \quad u_{m}(x) \leqslant u_{M}(x) .
$$

Since $\nabla^{2}\left(u_{M}-u_{m}\right)+\lambda\left[f\left(u_{M}\right)-f\left(u_{m}\right)\right]=0$, it follows from the mean value theorem that there exists a function $w_{0}$ such that:

(a) $(\forall x \in \bar{\Omega}) \quad u_{m}(x) \leqslant w_{0}(x) \leqslant u_{M}(x)$;

(b) the function $f^{\prime}\left(w_{0}\right)$ is continuous;

(c) there is a positive function $\varphi$ which satisfies

$$
\begin{aligned}
\nabla^{2} \varphi+\lambda f^{\prime}\left(w_{0}\right) \varphi & =0 & & \text { in } \Omega, \\
\varphi & =0 & & \text { on } \partial \Omega,
\end{aligned}
$$

Now, for $\lambda$ sufficiently large and $s \in(0, X]$, the open set $\Omega_{s}=\{x \in \Omega$ : $\left.d(x, \partial \Omega)>s / \lambda^{1 / 2}\right\}$ has a $C^{2+\sigma}$ boundary $S_{s}=\left\{x \in \bar{\Omega}: d(x, \partial \Omega)=s / \lambda^{1 / 2}\right\}$. Then define a function $I:[0, X] \rightarrow \mathbf{R}$ by

$$
I(s)=\int_{S_{s}} \varphi d S
$$

where $\varphi$ is a positive solution of (30) and $X$ is as found in Lemma 3. The function $I$ then satisfies the differential equation given in the following Proposition.

Proposition 4. If $\lambda$ is sufficiently large then $I$ is a $C^{2}$ function and

$$
\frac{d}{d s}\left(\frac{d I}{d s}+\frac{a_{2}(s)}{\lambda^{1 / 2}} I\right)+\left(f^{\prime}(z(s))+\frac{a_{1}(s)}{\lambda^{1 / 2}}\right) I=0, \quad s \in(0, X),
$$

where $a_{2}$ is a $C^{1}$ function. Further, there exist constants $A_{1}, A_{2}$ (independent of $\lambda$ ) such that

$$
(\forall s \in(0, X]) \quad\left|a_{1}(s)\right| \leqslant A_{1},\left|a_{2}(s)\right| \leqslant A_{2}
$$


Proof. Now, for $s \in[0, X]$ and $x \in S_{s}$, let $\mathbf{n}(x)$ denote the unit normal vector to the surface $S_{s}$ at the point $x$. Since $S_{s}$ is of class $C^{2+\sigma}$, the function $\mathbf{n}$ is of class $C^{2+\sigma}$. Then $\mathrm{n}$ can be extended to the whole of $\bar{\Omega}$ so that it remains a $C^{2+\sigma}$ function. Consequently, making use of the various forms of the divergence theorem, we have:

$$
\begin{aligned}
I(s) & =\int_{S_{s}}(\varphi \mathbf{n} \cdot \mathbf{n}) d S \\
& =\int_{\Omega_{s}} \nabla \cdot(\varphi \mathbf{n}) d x \\
& =\int_{\Omega_{s}}[(\nabla \varphi) \cdot \mathbf{n}+\varphi(\nabla \cdot \mathbf{n})] d x .
\end{aligned}
$$

Then, using the fact that, on $S_{s}, s=\lambda^{1 / 2} d(x, \partial \Omega)$,

$$
\begin{aligned}
\frac{d I}{d s} & =\frac{d}{d s} \int_{\Omega_{s}}[\nabla \varphi \cdot \mathbf{n}+\varphi(\nabla \cdot \mathbf{n})] d x \\
& =-\lambda^{-1 / 2} \int_{S_{s}}[\nabla \varphi \cdot \mathbf{n}+\varphi(\nabla \cdot \mathbf{n})] d S \\
& =\lambda^{-1 / 2} \int_{S_{s}} \frac{\partial \varphi}{\partial n} d S-\lambda^{-1 / 2} \int_{S_{s}} \varphi(\nabla \cdot \mathbf{n}) d S .
\end{aligned}
$$

Also, using (30), and the divergence theorem again

$$
\int_{S_{s}} \frac{\partial \varphi}{\partial n} d S=\int_{\Omega_{s}} \nabla^{2} \varphi d x=\int_{\Omega_{s}}-\lambda f^{\prime}\left(w_{0}\right) \varphi d x
$$

and so by considering $\int_{S_{s+h}}(\partial \varphi / \partial n) d S-\int_{S_{s}}(\partial \varphi / \partial n) d S$ we obtain

$$
\frac{d}{d s} \int_{S_{s}} \frac{\partial \varphi}{\partial n} d S=\lambda^{1 / 2} \int_{S_{s}} f^{\prime}\left(w_{0}\right) \varphi d S .
$$

Therefore using (31) we obtain

$$
\frac{d}{d s}\left[\frac{d I}{d s}+\lambda^{-1 / 2} \int_{S_{s}} \varphi(\nabla \cdot \mathbf{n}) d S\right]=-\int_{S_{s}} f^{\prime}\left(w_{0}\right) \varphi d S .
$$

Since $(\forall x \in \bar{\Omega}) 0 \leqslant u_{m}(x) \leqslant w_{0}(x) \leqslant u_{M}(x) \leqslant 1, f^{\prime}\left(w_{0}\right)$ is bounded on $\Omega$. In particular, for $s \in(0, X]$, there exist $x_{1}, x_{2}, \in S_{s}$ such that

$$
\left(\forall x \in S_{s}\right) \quad f^{\prime}\left(w_{0}\left(x_{1}\right)\right) \leqslant f^{\prime}\left(w_{0}(x)\right) \leqslant f^{\prime}\left(w_{0}\left(x_{2}\right)\right) .
$$

Thus, since $\varphi$ is positive on $\Omega$, we have, for $s>0$,

$$
f^{\prime}\left(w_{0}\left(x_{1}\right)\right) \leqslant\left(\int_{S_{s}} f^{\prime}\left(w_{0}\right) \varphi d S\right) /\left(\int_{S_{s}} \varphi d S\right) \leqslant f^{\prime}\left(w_{0}\left(x_{2}\right)\right) .
$$


If $\lambda$ is sufficiently large then Theorem 2 holds and for $x \in S_{s}, d(x, \partial \Omega)=s / \lambda^{1 / 2}$ $\leqslant \rho / 2$. Since $u_{m}(x)$ and $u_{M}(x)$ satisfy $(28)$,

$$
z(s)-B / \lambda^{1 / 2} \leqslant w_{0}(x) \leqslant z(s)+B / \lambda^{1 / 2} .
$$

Consequently, as $f^{\prime \prime}$ is bounded on $[0,1]$, there exists a constant $A_{1}$ such that, for $i=1,2$,

$$
\left|f^{\prime}(z(s))-f^{\prime}\left(w_{0}\left(x_{1}\right)\right)\right| \leqslant A_{1} / \lambda^{1 / 2} .
$$

Let $a_{1}(s)=\lambda^{1 / 2}\left(\int_{S_{s}} f^{\prime}\left(w_{0}\right) \varphi d S /\left(\int_{S_{s}} \varphi d S\right)-f^{\prime}(z(s))\right)$; then $\left|a_{1}(s)\right| \leqslant A_{1}$ and $a_{1}$ is a $C^{1}$ function. Then $\int_{S_{s}} f^{\prime}\left(w_{0}\right) \varphi d S=\left[f^{\prime}(z(s))+a_{1}(s) / \lambda^{1 / 2}\right] I(s)$. Similar arguments show that

$$
\int_{S_{s}} \varphi(\nabla \cdot \mathbf{n}) d S=a_{2}(s) I(s),
$$

where $a_{2}$ is a $C^{1}$ function and, for $s \in(0, X]$

$$
\left|a_{2}(s)\right| \leqslant A_{2}
$$

( $A_{2}$ is independent of $\lambda$ ). Therefore

$$
\frac{d}{d s}\left(\frac{d I}{d s}+a_{2}(s) I / \lambda^{1 / 2}\right)+\left(f^{\prime}(z(s))+a_{1}(s) / \lambda^{1 / 2}\right) I=0, \quad s \in(0, X) .
$$

Now, if $\varphi$ is a solution of (30) which is positive on $\Omega$ then the above result shows that $I^{\prime}(0)>0$. Hence, it is possible to choose such a $\varphi$ so that $I$ satisfies the differential equation of Proposition 4 with

$$
I(0)=0, \quad I^{\prime}(0)=1,
$$

and further, $(\forall s>0) I(s)>0$.

Lemma 5. Suppose $\lambda$ is sufficiently large and $I$ is the unique solution of the differential equation in Proposition 4 with the initial conditions

$$
I(0)=0, \quad I^{\prime}(0)=1
$$

Then, there exist constants $\Gamma, \Gamma_{1}$ (independent of $\lambda$ ) such that

$$
\begin{array}{ll}
(\forall s \in[0, X]) \quad I(s) \leqslant \Gamma \\
I^{\prime}(s) \leqslant \Gamma_{1} .
\end{array}
$$

Proof. Now, I satisfies the integro-differential equation

$$
\frac{d I}{d s}+\left(a_{2}(s) / \sqrt{\lambda}\right) I(s)=-\int_{0}^{s}\left(f^{\prime}(z(t))+a_{1}(t) / \sqrt{\lambda}\right) I(t) d t+1
$$

with $I(0)=0$. Since $0 \leqslant z \leqslant 1$ on $\mathbf{R}$, there exists a constant $M_{1}>1$ such that

$$
(\forall s \in \mathbf{R}) \quad\left|f^{\prime}(z(s))\right|<M_{1} \text {. }
$$


We now show that, for $\lambda$ sufficiently large,

$$
(\forall s \in[0, X]) \quad 0 \leqslant I(s)<e^{m_{1} s}
$$

where $m_{1}=\left(1+\sqrt{5+4 M_{1}}\right) / 2$.

Let $I_{1}(s)=e^{m_{1} s}$, since $m_{1}^{2}-m_{1}-\left(1+M_{1}\right)=0$ we have

$$
I_{1}^{\prime}-I_{1}=\left(1+M_{\mathrm{i}}\right) \int_{0}^{s} I_{\mathrm{i}}(t) d t+m_{\mathrm{i}}-1
$$

also

$$
I_{1}(0)=1
$$

and

$$
I_{1}^{\prime}(0)=m_{1}>2 .
$$

Consequently $I_{1}(s)>I(s)$ for $s$ in some neighbourhood of 0 . Suppose there exists a point $S \in(0, X]$ such that

$$
(\forall s \in[0,)) \quad I(s)<I_{1}(s) \text { and } I(S)=I_{1}(S) \text {; }
$$

then, since $I(s)>0$ for $s \in(0, X], I^{\prime}(S) \geqslant I_{1}^{\prime}(S)$. However, from (33), if $\lambda$ is sufficiently large,

$$
\begin{aligned}
I^{\prime}(S) & =-\left(a_{2}(S) / \sqrt{\lambda}\right) I(S)-\int_{0}^{S}\left[f^{\prime}(z(t))+a_{1}(t) / \sqrt{\lambda}\right] I(t) d t+1 \\
& <\left(A_{2} / \sqrt{\lambda}\right) I_{1}(S)+\int_{0}^{S}\left(M_{1}+A_{1} / \sqrt{\lambda}\right) I_{1}(t) d t+m_{1}-1 \\
& <I_{1}(S)+\left(M_{1}+1\right) \int_{0}^{S} I_{1}(t) d t+m_{1}-1 \\
& =I_{1}^{\prime}(S) .
\end{aligned}
$$

This contradiction means that

$$
(\forall s \in[0, X]) \quad I(s)<I_{1}(s)=e^{m_{1} s} .
$$

The first inequality in the lemma follows by taking $\Gamma=\exp \left(m_{1} X\right)$. The second inequality can then be easily derived from (33).

Using these last three results we can show that the function $I$ tends to the solution of (29) as $\lambda \rightarrow \infty$. In particular we have:

Proposition 6. There exists a constant $L>0$ such that, for $\lambda$ sufficiently large,

$$
\frac{d I}{d s}(X) \geqslant L
$$


Proof. From (29) and (33) we see that, for $\lambda$ sufficiently large,

$$
\begin{aligned}
\frac{d}{d s}(\psi & -I)+\int_{0}^{s} f^{\prime}(z(t))(\psi(t)-I(t)) d t \\
& =\left(a_{2}(s) / \sqrt{\lambda}\right) I(s)-\int_{0}^{s}\left(a_{1}(t) / \sqrt{\lambda}\right) I(t) d t
\end{aligned}
$$

with $\psi(0)-I(0)=0$.

Letting $J=\psi-I$ and $J_{1}(s)=\left(M / \sqrt{\lambda M_{1}}\right) \exp \left(\sqrt{M_{1}} s\right)$, where $M=$ $\left(A_{2}+X A_{1}\right) \Gamma$ and $M_{1}$ is an upper bound for $\left|f^{\prime}(z)\right|$ as given in Lemma 6, similar arguments to those of Lemma 5 show that, for $\lambda$ sufficiently large

$$
(\forall s \in[0, X]) \quad J(s) \leqslant J_{1}(s) \text {. }
$$

Therefore $J(s) \rightarrow 0$ uniformly on $[0, X]$ as $\lambda \rightarrow \infty$; and so $I(s) \rightarrow \psi(s)$ uniformly on $[0, X]$ as $\lambda \rightarrow \infty$.

Using this and the boundedness of $a_{1}, a_{2}$ and $I$, it follows from (34) that

$$
(\forall s \in[0, X]) \quad \frac{d I}{d s}(s) \rightarrow \frac{d \psi}{d s}(s) \quad \text { as } \lambda \rightarrow \infty .
$$

From Lemma 3 we have $(d \psi / d s)(X)>0$; so for $\lambda$ sufficiently large

$$
\frac{d I}{d s}(X)>\frac{d \psi}{d s}(X) / 2 \text {. }
$$

We shall use the equation that $\varphi$ satisfies and the inequalities of Theorem 2 to show that $(d I / d s)(X) \rightarrow l$ as $\lambda \rightarrow \infty$ and $l \leqslant 0$.

Now, suppose $x \in \Omega_{X}$ and $d(x, \partial \Omega)=s / \lambda^{1 / 2} \leqslant \rho / 2$; then, by Theorem 2 and the definition of $w_{0},\left|z(s)-w_{0}(x)\right|<B / \lambda^{1 / 2}$. Since $z([X, \infty)) \subseteq(0,1)$ and $f^{\prime}$ is continuous, it follows that there exists $\delta>0$ such that

$$
(\forall t \in[X, \infty)) \quad|z(t)-r|<\delta \Rightarrow\left|f^{\prime}(z(t))-f^{\prime}(r)\right|<\Pi_{1} / 2 .
$$

In particular $(\forall t \in[X, \infty))|z(t)-r|<\delta \Rightarrow f^{\prime}(r)<0$. So, if $\lambda$ is sufficiently large then $\left|z(s)-w_{0}(x)\right|<\delta$ and $f^{\prime}\left(w_{0}(x)\right)<0$. That is, for $\lambda$ sufficiently large, if $x \in \Omega_{X}$ with $d(x, \partial \Omega) \leqslant \rho / 2$ then $f^{\prime}\left(w_{0}(x)\right)<0$.

It follows from Theorem 2 that, for $x \in \Omega_{X}$ with $d(x, \partial \Omega) \geqslant \rho / 2$, we have

$$
1-\exp \left(-A \lambda^{1 / 2}\right)<w_{0}(x) \leqslant 1 \text {. }
$$

Since $f^{\prime}(1)<0$, it follows that for $\lambda$ sufficiently large we have, for any $x \in \Omega_{X}$ with $d(x, \partial \Omega) \geqslant \rho / 2$,

$$
f^{\prime}\left(w_{0}(x)\right)<0 .
$$

Consequently we see that for $\lambda$ sufficiently large,

$$
\left(\forall x \in \Omega_{X}\right) \quad f^{\prime}\left(w_{0}(x)\right)<0 .
$$

Therefore, by (32),

$$
\int_{S_{X}} \frac{\partial \varphi}{\partial n} d S=-\lambda \int_{\Omega_{X}} f^{\prime}\left(w_{0}\right) \varphi d x>0
$$


and by (31)

$$
\begin{aligned}
\int_{S_{X}} \frac{\partial \varphi}{\partial n} d S & =-\lambda^{1 / 2} \frac{d I}{d s}(X)-\int_{S_{X}} \varphi(\nabla \cdot \mathbf{n}) d S \\
& =-\lambda^{1 / 2}\left[\frac{d I}{d s}(X)+\lambda^{-1 / 2} a_{2}(X) I(X)\right] .
\end{aligned}
$$

Consequently, we either have

(a) for suîficientiy iarge $\lambda,(d i \bar{I} / \dot{A})(X)<\hat{0}$; or

(b) $(d I / d s)(X) \rightarrow 0$ as $\lambda \rightarrow \infty$.

In either case we have a contradiction of the result in Proposition 6.

Hence the assumption that (21) has at least two solutions for arbitrarily large values of $\lambda$ was false. That is (21) has a unique solution for sufficiently large $\lambda$.

\section{Uniqueness of solutions for large $\alpha$}

In this section we shall show that the equations (1), (2) have a unique solution for all values of $\lambda$ if $\alpha$ is large enough. Of course we have to exclude the case $\mu<\nu=\infty$, since we showed in Part II (Burnell, Lacey and Wake [2]) that (1), (2) always has multiple solutions for any value of $\alpha$, if $\mu\langle\nu=\infty$ and $n=1$.

Firstly we note that (1), (2) have a solution if and only if the equations

$$
\begin{array}{cl}
\nabla^{2} v-\lambda \alpha(1+v) e^{(1 / \alpha)(h-v)}=0 & \text { in } \Omega, \\
\frac{\partial v}{\partial n}+\nu v=0 & \text { on } \partial \Omega, \\
\nabla^{2} h=0 & \text { in } \Omega, \\
\frac{\partial h}{\partial n}+\mu h=(\mu-\nu) v & \text { on } \partial \Omega,
\end{array}
$$

have a solution. This follows since $(u, v)$ satisfy (1), (2) if and only if $(v, \alpha u+v)$ satisfy (35), (36). Thus we need only show that (35), (36) has a unique solution for $\alpha$ sufficiently large.

Define a map $S: C(\bar{\Omega}) \times C(\bar{\Omega}) \rightarrow C(\bar{\Omega}) \times C(\bar{\Omega})$ as follows: $S(v, h)=\left(v^{\prime}, h^{\prime}\right)$ where $v^{\prime}, h^{\prime}$ are the unique solutions of the equations

$$
\begin{array}{cc}
\nabla^{2} v^{\prime}-\lambda \alpha v^{\prime}=\lambda \alpha(1+v) e^{(1 / \alpha)(h-v)}-\lambda \alpha v & \text { in } \Omega, \\
\frac{\partial v^{\prime}}{\partial n}+\nu v^{\prime}=0 & \text { on } \partial \Omega,
\end{array}
$$

and

$$
\begin{array}{cl}
\nabla^{2} h^{\prime}=0 & \text { in } \Omega, \\
\frac{\partial h^{\prime}}{\partial n}+\mu h^{\prime}=(\mu-\nu) v^{\prime} & \text { on } \partial \Omega,
\end{array}
$$


respectively. Clearly $(v, h)$ is a fixed point of $S$ if and only if $(v, h)$ is a solution of (35), (36). Further, it follows from Section 1, Theorem 1 and the maximum principle that if $(v, h)$ is a fixed point of $S$ then

$$
\begin{gathered}
(\forall x \in \bar{\Omega}) \quad-1 \leqslant v(x) \leqslant 0, \\
0 \leqslant h(x) \leqslant \nu / \mu-1 \quad \text { if } \mu<\nu, \\
-1+\nu / \mu \leqslant h(x) \leqslant 0 \quad \text { if } \mu \geqslant \nu .
\end{gathered}
$$

We prove that (1), (2) have a unique solution for large $\alpha$ by showing that two fixed points of $S$ must satisfy a certain inequality. We derive this inequality from the following result.

Proposition 1. There exists a constant $A$ (independent of $\lambda, \alpha$ ) such that if:

(a) $\alpha>\max \{1, \nu / \mu\}$

(b) $v_{1}, v_{2}, h_{1}, h_{2} \in C(\bar{\Omega})$ and, for $i=1,2$,

$$
\begin{array}{cl}
(\forall x \in \bar{\Omega}) \quad-1 \leqslant v_{i}(x) \leqslant 0, & \\
0 \leqslant h_{i}(x) \leqslant \nu / \mu-1 & \text { if } \mu<\nu, \\
-1+\nu / \mu \leqslant h_{i}(x) \leqslant 0 & \text { if } \mu \geqslant \nu .
\end{array}
$$

and

(c) $\left(v_{1}^{\prime}, h_{1}^{\prime}\right)=S\left(v_{1}, h_{1}\right), \quad\left(v_{2}^{\prime}, h_{2}^{\prime}\right)=S\left(v_{2}, h_{2}\right)$;

then

$$
\left\|v_{1}^{\prime}-v_{2}^{\prime}\right\|_{0}+\left\|h_{2}^{\prime}-h_{2}^{\prime}\right\|_{0} \leqslant(1 / \alpha) A\left[\left\|v_{1}-v_{2}\right\|_{0}+\left\|h_{1}-h_{2}\right\|_{0}\right] \text {. }
$$

Proof. Now, from the definition of $S$ we have

$$
\begin{aligned}
& \nabla^{2}\left(v_{1}^{\prime}-v_{2}^{\prime}\right)-\lambda \alpha\left(v_{1}^{\prime}-v_{2}^{\prime}\right) \\
& =\lambda \alpha\left[e^{(1 / \alpha)\left(h_{1}-v_{1}\right)}-e^{(1 / \alpha)\left(h_{2}-v_{2}\right)}+v_{1}\left(e^{(1 / \alpha)\left(h_{1}-v_{1}\right)}-e^{(1 / \alpha)\left(h_{2}-v_{2}\right)}\right)\right. \\
& \left.+\left(v_{1}-v_{2}\right) e^{(1 / \alpha)\left(h_{2}-v_{2}\right)}-\left(v_{1}-v_{2}\right)\right] \\
& =\lambda \alpha\left[\left(1+v_{1}\right)\left(e^{(1 / \alpha)\left(h_{1}-v_{1}\right)}-e^{(1 / \alpha)\left(h_{2}-v_{2}\right)}\right)\right. \\
& \left.+\left(v_{1}-v_{2}\right)\left(e^{(1 / \alpha)\left(h_{2}-v_{2}\right)}-1\right)\right] \\
& =\lambda \alpha\left[(1 / \alpha)\left(1+v_{1}\right) e^{\xi}\left(h_{1}-h_{2}+v_{2}-v_{1}\right)\right. \\
& \left.+\left(v_{1}-v_{2}\right)\left(e^{(1 / \alpha)\left(h_{2}-v_{2}\right)}-1\right)\right]
\end{aligned}
$$

where $\xi$ is a function with $\xi(x)$ lying between $(1 / \alpha)\left(h_{1}(x)-v_{1}(x)\right)$ and $(1 / \alpha)\left(h_{2}(x)-v_{2}(x)\right)$, for all $x \in \bar{\Omega}$. Since, for $i=1,2,(1 / \alpha)\left(h_{1}-v_{i}\right)<\nu / \alpha \mu$ if $\mu<\nu$, and $(1 / \alpha)\left(h_{i}-v_{i}\right)<1 / \alpha$ if $\mu \geqslant \nu$, we have

$$
\begin{aligned}
& \left|\lambda \alpha\left[(1 / \alpha)\left(1+v_{1}\right) e^{\xi}\left(h_{1}-h_{2}+v_{2}-v_{1}\right)+\left(v_{1}-v_{2}\right)\left(e^{(1 / \alpha)\left(h_{2}-v_{2}\right)}-1\right)\right]\right| \\
& \leqslant \lambda \alpha\left\{(1 / \alpha)\left|1+v_{1}\right| e^{\xi}\left(\left|h_{1}-h_{2}\right|+\left|v_{2}-v_{1}\right|\right)+\left|v_{1}-v_{2}\right|\left|e^{(1 / \alpha)\left(h_{2}-v_{2}\right)}-1\right|\right\} \\
& \leqslant \lambda \alpha\left\{(1 / \alpha) e^{\beta}\left\|h_{1}-h_{2}\right\|_{0}+\left((1 / \alpha) e^{\beta}+\left|e^{\beta}-1\right|\right)\left\|v_{1}-v_{2}\right\|_{0}\right\},
\end{aligned}
$$

where $\beta=\max \{1 / \alpha, \nu / \alpha \mu\}$. 
Noting that $\left|e^{\beta}-1\right|<\beta+\left(\beta^{2} / 2\right) e^{\beta}$, and applying the maximum principle we see that

$$
\begin{aligned}
&\left\|v_{1}^{\prime}-v_{2}^{\prime}\right\|_{0} \leqslant(1 / \lambda \alpha)\left(\lambda \alpha \left\{(1 / \alpha) e^{\beta}\left\|h_{1}-h_{2}\right\|_{0}\right.\right. \\
&\left.\left.+\left((1 / \alpha) e^{\beta}+\beta+\left(\beta^{2} / 2\right) e^{\beta}\right)\left\|\left(v_{1}-v_{2}\right)\right\|_{0}\right\}\right) \\
&=(1 / \alpha) e^{\beta}\left\|h_{1}-h_{2}\right\|_{0}+\left((1 / \alpha) e^{\beta}+\beta+\left(\beta^{2} / 2\right) e^{\beta}\right)\left\|_{1}-v_{2}\right\|_{0} .
\end{aligned}
$$

Áiso,

$$
\begin{aligned}
\nabla^{2}\left(h_{1}^{\prime}-h_{2}^{\prime}\right) & =0 \text { in } \Omega, \\
\frac{\partial\left(h_{1}^{\prime}-h_{2}^{\prime}\right)}{\partial n}+\mu\left(h_{1}^{\prime}-h_{2}^{\prime}\right) & =(\mu-\nu)\left(v_{1}^{\prime}-v_{2}^{\prime}\right) \text { on } \partial \Omega,
\end{aligned}
$$

where we note that if $\nu<\mu=\infty$ this boundary condition becomes

$$
h_{1}^{\prime}-h_{2}^{\prime}=v_{1}^{\prime}-v_{2}^{\prime} \text { on } \partial \Omega .
$$

Hence it follows from the maximum principle that

$$
\left\|h_{1}^{\prime}-h_{2}^{\prime}\right\|_{0} \leqslant l(\mu, \nu)\left\|v_{1}^{\prime}-v_{2}^{\prime}\right\|_{0}
$$

where $l(\mu, \nu)=|\mu-\nu| / \mu$ if $\mu<\infty$, and $l(\mu, \nu)=1$ if $\mu=\infty$. Consequently

$$
\begin{aligned}
& \left\|v_{1}^{\prime}-v_{2}^{\prime}\right\|_{0}+\left\|h_{1}^{\prime}-h_{2}^{\prime}\right\|_{0} \\
& \leqslant(1+l(\mu, \nu))\left\{(1 / \alpha) e^{\beta}\left\|h_{1}-h_{2}\right\|_{0}\right. \\
& \left.\quad+\left((1 / \alpha) e^{\beta}+\beta+\left(\beta^{2} / 2\right) e^{\beta}\right)\left\|v_{1}-v_{2}\right\|_{0}\right\} \\
& \leqslant(1+l(\mu, \nu))\left((1 / \alpha) e^{\beta}+\beta+\left(\beta^{2} / 2\right) e^{\beta}\right)\left[\left\|h_{1}-h_{2}\right\|_{0}+\left\|v_{1}-v_{2}\right\|_{0}\right] .
\end{aligned}
$$

Since $\alpha>\max \{1, \nu / \mu\}$, we have if $\mu \geqslant \nu$,

$$
\begin{aligned}
& (1 / \alpha) e^{\beta}+\beta+\left(\beta^{2} / 2\right) e^{\beta} \\
& \quad=(1 / \alpha) e^{1 / \alpha}+(1 / \alpha)+\left(1 / 2 \alpha^{2}\right) e^{1 / \alpha}<(1 / \alpha)\left[e+1+\frac{1}{2} e\right]
\end{aligned}
$$

and if $\mu<\nu$,

$$
\begin{aligned}
(1 / \alpha) e^{\beta}+\beta+\left(\beta^{2} / 2\right) e^{\beta} & =(1 / \alpha) e^{\nu / \alpha \mu}+(\nu / \alpha \mu)+\frac{1}{2}\left(\nu^{2} / \alpha^{2} \mu^{2}\right) e^{\nu / \alpha \mu} \\
& <(1 / \alpha)\left[e+\nu / \mu+\frac{1}{2}(\nu / \mu) e\right] .
\end{aligned}
$$

Therefore the result follows by taking

$$
\begin{aligned}
A & =(1+l(\mu, \nu))\left(1+\frac{3}{2} e\right) \quad \text { if } \mu \geqslant \nu, \\
& =(1+l(\mu, \nu))\left(\nu / \mu+\left(1+\frac{1}{2} \nu / \mu\right) e\right) \quad \text { if } \mu<\nu . \quad \text { Q.E.D. }
\end{aligned}
$$

THeorem 2. If $\alpha$ is sufficiently large then (1), (2) have a unique solution for all values of $\lambda$.

Proof. Let $A$ be as given in Proposition 1; and suppose $\alpha>\max \{1, \nu / \mu, A\}$. If $\left(u_{1}, v_{1}\right),\left(u_{2}, v_{2}\right)$ are solutions of (1), (2) for this value of $\alpha$ and any value of $\lambda$ 
then $\left(v_{1}, h_{1}\right),\left(v_{2}, h_{2}\right)$ are fixed points of $S$, where $h_{1}=\alpha u_{1}+v_{1}, h_{2}=\alpha u_{2}+v_{2}$. Therefore, by Proposition 1,

$$
\begin{aligned}
\left\|h_{1}-h_{2}\right\|_{0}+\left\|v_{1}-v_{2}\right\|_{0} & \leqslant(1 / \alpha) A\left[\left\|v_{1}-v_{2}\right\|_{0}+\left\|h_{1}-h_{2}\right\|_{0}\right] \\
& <\left\|v_{1}-v_{2}\right\|_{0}+\left\|h_{1}-h_{2}\right\|_{0} .
\end{aligned}
$$

Clearly this inequality can only be satisfied if $v_{1}=v_{2}$ and $h_{1}=h_{2}$, and hence $u_{1}=u_{2}$. Consequently (1), (2) has a unique solution for any value of $\lambda$ if $\alpha>\max \{1, \nu / \mu, A\}$.

Q.E.D.

\section{Bounds on the region of multiplicity}

Implicit in all the proofs of these results on the uniqueness and nonuniqueness of solutions of (1), (2), given here and in Part II (Burnell, Lacey and Wake [2]), are estimates of the range of values of $\lambda$ and $\alpha$ for which (1), (2) has multiple solutions. Here we shall state the results that follow from these proofs. Unfortunately, these estimates are rather crude as we made no effort in the proofs to find the best values of the parameters above (or below) which there is a unique (or multiple) solution. However we felt that it would be useful to record all these estimates. Once again we exclude the case $\mu<\nu=\infty$.

For any value of $\alpha$ let $\lambda_{b}$ be the infimum of the set of values of $\lambda$ for which (1), (2) has at least two solutions; and let $\lambda^{b}$ denote the supremum of this set. Also, let $K_{\mu}, K_{\nu}$ be the maps defined in Section 2, Theorem 1, and $\left\|K_{\mu}\right\|_{0},\left\|K_{\nu}\right\|_{0}$ be their operator norms.

THEOREM 1. (a) $\lambda_{b} \geqslant e^{-1 / \alpha} /\left(\left\|K_{\mu}\right\|_{0}+\alpha\left\|K_{\nu}\right\|_{0}\right)$.

(b) If $\mu=\nu$ then $\lambda_{b} \geqslant e^{2-1 / \alpha} /\left(\alpha\left\|K_{\mu}\right\|_{0}\right)$.

(c) If $\alpha$ is sufficiently small then

$$
\lambda_{b} \leqslant \frac{\nu / \mu+\sqrt{\nu^{2} / \mu^{2}-4 \alpha \nu / \mu}}{\nu / \mu-\sqrt{\nu^{2} / \mu^{2}-4 \alpha \nu / \mu}} \exp \left\{-\left(\nu / \mu+\sqrt{\nu^{2} / \mu^{2}-4 \alpha \nu / \mu}\right) / 2 \alpha\right\}\left\|\frac{f}{w}\right\|_{0},
$$

where $f: \bar{\Omega} \rightarrow \mathbf{R}$ is a $C^{1}$ function with support in $\Omega$ and $w$ is the unique solution of $\nabla^{2} w+f=0$ in $\Omega, \partial w / \partial n+\mu w=0$ on $\partial \Omega$.

(d) If $\lambda_{0}$ is the supremum of the set of values of $\lambda$ for which the equation

$$
\left.\begin{array}{ll}
\nabla^{2} u+\lambda e^{u}=0 & \text { in } \Omega, \\
\frac{\partial u}{\partial n}+\mu u=0 & \text { on } \Omega,
\end{array}\right\}
$$

has a solution and $\alpha$ is sufficiently small, then $\lambda^{b} \geqslant \lambda_{0}$. 
(e) If $\alpha$ is sufficiently small then

$$
\lambda^{b} \leqslant \max \left\{\frac{4(2-\nu / \mu) A e^{(1-\nu / \mu) / \alpha}}{\alpha^{3}}, \frac{1}{k^{2} \alpha\left(1-\left(\left\|\nabla w_{1}\right\|_{0}\right)^{2}\right)^{2}}\right\},
$$

where $w_{1}$ satisfies

$$
\begin{aligned}
\nabla^{2} \ddot{w}_{1}+1 / \hat{k}=\hat{0} & \text { in } \Omega, \\
w_{1}=0 & \text { on } \partial \Omega,
\end{aligned}
$$

with $k>1$ and $\left\|\nabla w_{1}\right\|_{0}<1, A=\nu / \min _{x \in \partial \Omega}\left\{-\left(\partial w_{1} / \partial n\right)(x)\right\}$.

\section{References}

[1] J. G. Burnell, A. A. Lacey and G. C. Wake, "Steady states of the reaction-diffusion equations. Part I: Questions of existence and continuity of solution branches", J. Austral. Math. Soc. Ser. B 24 (1983), 374-391.

[2] J. G. Burnell, A. A. Lacey and G. C. Wake, "Steady states of the reaction-diffusion equations. Part II: Uniqueness of solutions and some special cases", J. Austral. Math. Soc. Ser. B 24 (1983), 392-416.

[3] H: B. Keller and D. S. Cohen, "Some positone problems suggested by nonlinear heat generation", J. Math. Mech. 16 (1967), 1361-1376. 